\title{
Evolución del currículo médico y los nuevos desafíos
}

\section{Evolution of the medical curriculum and new challenges}

\author{
Camilo Torres Serna ${ }^{1}$
}

\section{RESUMEN}

Este artículo describe la influencia del Informe Flexner de 1910 sobre las universidades colombianas. Plantea la problemática actual de formación de médicos generales y su satisfacción académica, social y laboral. Invita a reflexionar sobre la necesidad de introducir cambios en el currículo universitario.

Palabras claves: currículo, médico general, informe Flexner, educación médica. (DeCS)

\begin{abstract}
This article describes the evolution of the medical curriculum under the Flexner Report of 1910 and its influence on Colombian universities. The current problem of training of general practitioners and their academic, social and work satisfaction is raised. The article invites us to reflect on the need to introduce changes in the university curriculum.
\end{abstract}

Keywords: medical curriculum, general practitioner, Flexner report, medical education. (MeSH)

\section{Historial del artículo:}

Fecha de recepción: 09/04/2018

Fecha de aceptación: 12/12/2018

1 Universidad del Valle, Universidad Santiago de Cali. Magister en Farmacología, Magister en Salud Pública, Magister en Dirección Universitaria, Doctor en Ciencias de la Educación. Cali, Colombia.

Correspondencia: Camilo Torres Serna, Dirección: Ciudad Universitaria Meléndez Calle 13 \# 100-00, Sede San Fernando Calle 4B \# 36-00 Universidad del Valle, Cali, Colombia, Teléfono: (2) 33958 31. Correo electrónico: catorse@yahoo.com

Como citar este artículo: Torres-Serna C. Evolución del currículo médico y los nuevos desafíos. Revista de la Facultad de Ciencias de la Salud de la Universidad del Cauca. 2019; 21 (1):34-42 


\section{INTRODUCCIÓN}

En la historia de la humanidad, siempre han existido personas dedicadas a cuidar de la salud de sus congéneres. Brujos, magos, sanadores, chamanes, etc. han ejercido empíricamente la medicina desde el origen del hombre hasta hoy (1).

La formación de médicos se fue dando por la trasmisión ancestral y directa de un sanador experimentado al aprendiz. Y así se inició también la formación formal, el estudiante ingresaba a un hospital y con la tutoría de un médico experimentado comenzaba a evaluar enfermos, su cuadro clínico y sus opciones de manejo en búsqueda de la recuperación de la salud (2).

Este enfoque académico estaba basado en el desarrollo de una buena historia clínica y un buen examen físico, aspectos fundamentales del denominado popularmente "ojo clínico". Según la inquietud personal de cada estudiante se revisaban las causas de la enfermedad y su base fisiopatológica (biología, bioquímica, fisiología etc.), temas de poco conocimiento en la época en que este modelo predominó (3).

La institucionalización de ese modelo muy utilizado en Europa en los siglos 16 y 17, se le conoció en Colombia como "medicina francesa” y predominó hasta mediados del siglo 20 (4).

A principios del siglo 20 científicos y filósofos naturales se unieron para formar un grupo de hombres que formaban lo que puede llamarse el Círculo Hopkins, su resultado fue un proyecto que alteró el curso de la educación médica en Estados Unidos. Ellos construyeron el edificio del sistema de educación médica que se conoce desde inicios del siglo 20 (5). Sus esfuerzos resultaron exitosos en la fundación basada en la ciencia de la formación médica que ha hecho de los Estados Unidos el líder reconocido en la educación médica y la investigación médica en la actualidad.

El grupo estaba conformado por un graduado de Yale, William Welch, quien fue en gran parte el creador intelectual de Hopkins. Otro miembro del grupo era Frederick Gates, un ministro bautista y asesor de confianza para John D. Rockefeller (6).

El tercer miembro del círculo fue Abraham Flexner, un maestro de escuela y experto en las prácticas educativas, cuyos antecedentes y formación lo convirtió en un caso atípico en el círculo (7).
Figura 1. Modelo Flexner para el currículo médico.

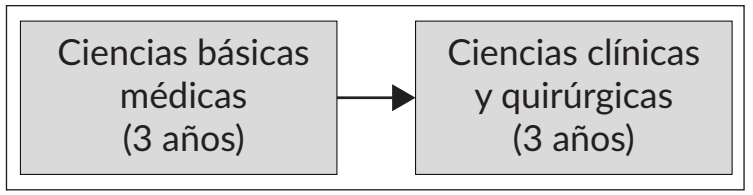

Fuente: Flexner, Medical Education in The Unites States and Canada, 1910 (14).

\section{PRINCIPIOS DEL SIGLO XX: EL INFORME FLEXNER}

Se reconoce a Flexner, con entera justicia, como el gran reformista, el gran transformador de la educación médica. Pero yendo más allá, algunos consideran que, con la sola excepción del Ratio Studiorum Jesuita y de la idea de universidad de Henry Newman, ningún pronunciamiento sobre filosofía educativa ha tenido tan decisiva y vasta influencia como el "Informe Flexner" de 1910 (8). Es imposible hablar de modificaciones al currículo de las escuelas de medicina sin revisitar a Flexner.

A lo largo de su vida, Flexner, fue un ardiente defensor del estilo pedagógico alemán de la educación médica y fue firme en su convicción de que la medicina era una disciplina científica que podría ser mejor realizada utilizando el modelo alemán como el prototipo en América (9-10).

Su informe a la Fundación Carnegie para el Avance de la Enseńanza, fue publicado en 1910 y en Colombia, su propuesta de reforma curricular fue impulsadas por Universidad del Valle (11-13).

\section{Época pos Flexner}

La primera modificación al modelo Flexner apareció con la creación de la OMS, después de la Segunda Guerra Mundial, ańo 1945. Las políticas de la OMS eran tendientes al desarrollo de la salud pública y sus componentes, epidemiología, promoción de la salud, prevención de la enfermedad, etc. Las facultades de medicina involucraron estos temas al modelo Flexneriano.

Desde alrededor de los ańos 70 hasta la fecha, varias facultades de medicina del mundo han hecho propuestas de estrategias diferentes de aplicación del modelo Flexneriano. 
Se destaca la Universidad de McMaster en Canada, que desarrolló de manera formal un modelo denominado aprendizaje basado en problemas (ABP), en el cual logró una mayor integración de las ciencias básicas médicas y las ciencias clínicas a través de la búsqueda de la solución a un problema médico específico (15).

Posteriormente, se desarrolló una corriente mundial para realizar el currículo médico basado en competencias, pero bajo el mismo modelo Flexneriano (ciencias básicas/ ciencias clínicas) (16-20).

Actualmente se plantean otras modificaciones al currículo médico, pero básicamente basadas en el modelo Flexner $(21,22)$.

\section{FIN DEL SIGLO XX Y COMIENZO DEL SIGLO XXI}

Publicaciones de la Universidad del Valle (23), de profesores colombianos Patińo (24) y de Ascofame, han planteado la necesidad de revisar el currículo médico (25-26).
En lo referente a las ciencias biológicas, cabe cuestionar la viabilidad de los departamentos tradicionales de ciencias básicas, como lo ha planteado Petersdorf, Presidente de la Asociación Americana de Escuelas de Medicina (AAMC): “¿Pueden persistir los seis grandes clásicos -anatomía, fisiología, patología, microbiología y farmacología- en un ambiente donde los anatomistas son cirujanos, donde la moderna histología y la bioquímica han sido superadas por la biología celular y molecular, donde la fisiología orgánica es enseńada por profesores de medicina interna, donde la neurociencia es una floreciente disciplina que comprende neurobiología, neurofisiología, neuroquímica, neuropatología y aun ciertos aspectos de la neurología clínica, y donde la farmacología se hace progresivamente más una ciencia molecular dejando la enseńanza de la terapéutica a los departamentos clínicos? En tal entorno, ¿no sería más racional enfocarse hacia menos departamentos de ciencias básicas, denominándolos de acuerdo con lo que realmente son: biología molecular y celular, neurociencia y fisiología orgánica, farmacología y patología, para constituirlos en el fundamento de la ciencia básica en nuestras escuelas médicas, ¿en reemplazo de los seis departamentos tradicionales?" (27-35).

Figura 2. Evolución histórica del currículo médico en todo el mundo.

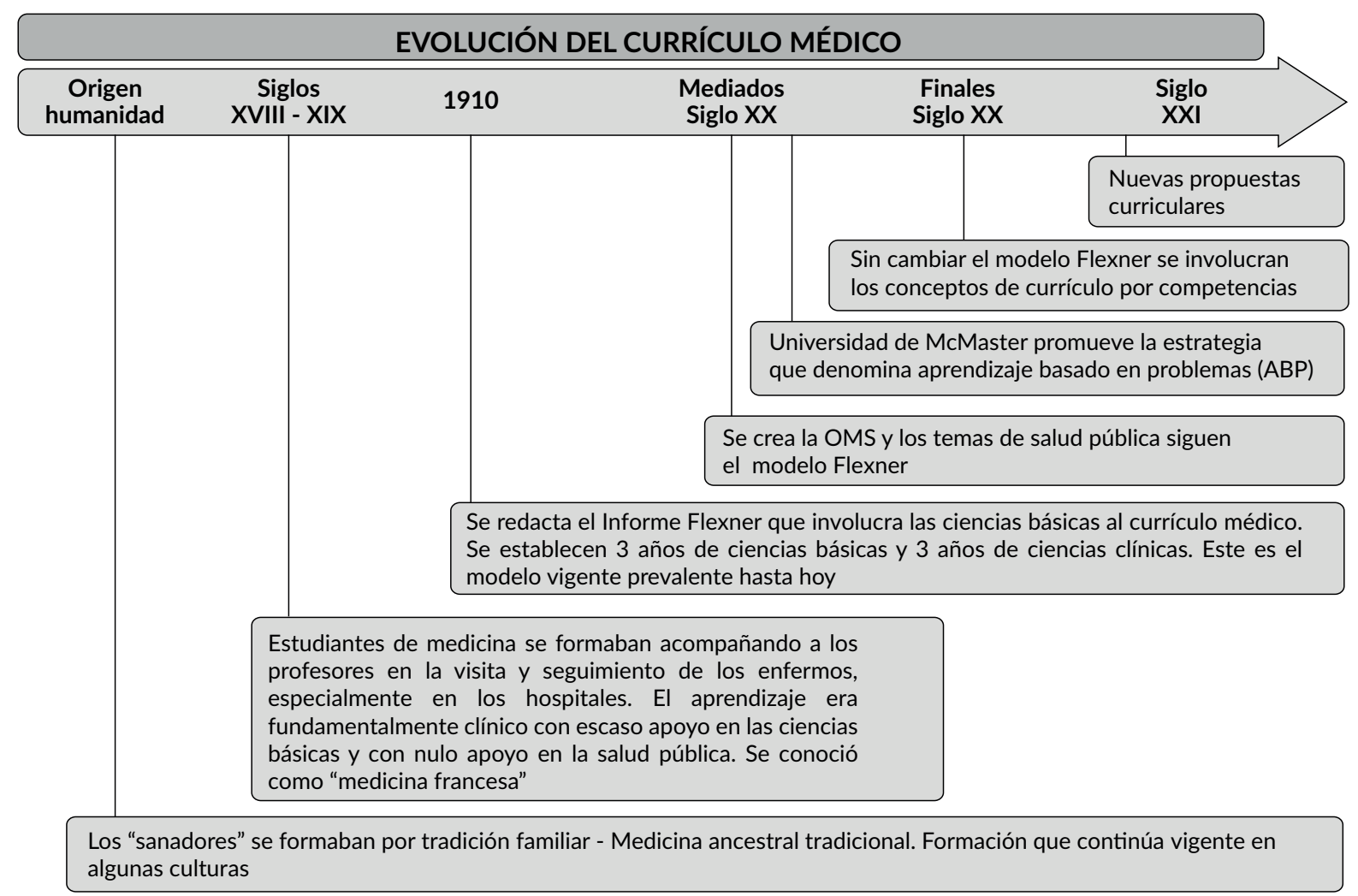

Fuente: elaboración propia basado en Lain (1), Serpa (3), Flexner (6). 
Muchas escuelas de medicina han estudiado la gran problemática de la formación de médicos. En la Universidad del Cauca, en Popayán, Colombia, se realizó una evaluación de los componentes de formación que idealmente el médico general debería adquirir, como son valores profesionales, actitudes y comportamiento ético, fundamentación científica, pensamiento lógico e investigativo, habilidades en la comunicación social, habilidades clínicas, salud de población y sistemas de salud y manejo de la información (36).

Dos propuestas internacionales adicionales se deben destacar:

El proyecto del Colegio Real de Médicos y Cirujanos de Canadá que desde el ańo 2000 impulsa a definir un esquema de competencias necesarias en la formación de un médico para cada entorno (37). El modelo desarrollado se conoce CanMEDS y en la actualidad sirve de base para los programas de formación de médicos de muchos países en todo el mundo generando un movimiento en la educación clínica hacia un enfoque basado en las competencias (38,39).

Otra propuesta novedosa ha sido planteada por Bligh que acuńo el acrónimo PRISMS para una nueva estrategia de desarrollo curricular que plantea que la formación del médico debe realizarse con un elevado componente Práctico, con temas Relevantes, de manera Interdisciplinaria, en cursos cortos (Shorter), en diferentes escenarios de atención clínica (Multicéntrico) y con el reconocimiento de la importancia del trabajo en equipo en salud (40).

\section{LA FORMACIÓN DE MÉDICOS EN COLOMBIA.}

Ser médico en Colombia sigue siendo una ilusión de cambio de forma de vida para muchos jóvenes y especialmente para sus familias. La sociedad ha dado al médico una categoría social especial y la familia se siente orgullosa de ello (41).

Esto ha llevado a que desde hace muchos ańos se plantee la discusión del tipo de médico que se debe formar $(42,43)$. Siempre hay una misma conclusión: no se está formando el médico general que se requiere. Es una verdad que se está camuflando con gran perjuicio para el sistema de salud:

- La ciencia ha crecido tanto (y sigue creciendo) que es imposible que un médico "general" sepa de todo como lo desean los expertos profesores que, curiosamente, solo saben de un tema.

- Nadie ingresa a estudiar medicina para ser médico general, todos los estudiantes tienen desde el primer día en la universidad el proyecto de vida de ser especialistas en un solo campo de la medicina (44-49).

- Los usuarios, pacientes, no quieren ser atendidos por un médico general (50-51).

El flujograma de los resultados del ejercicio profesional de médicos generales sin la suficiente capacitación aparece en la Figura 3.

Figura 3. Flujograma de resultados del ejercicio profesional de médicos generales sin la suficiente capacitación.

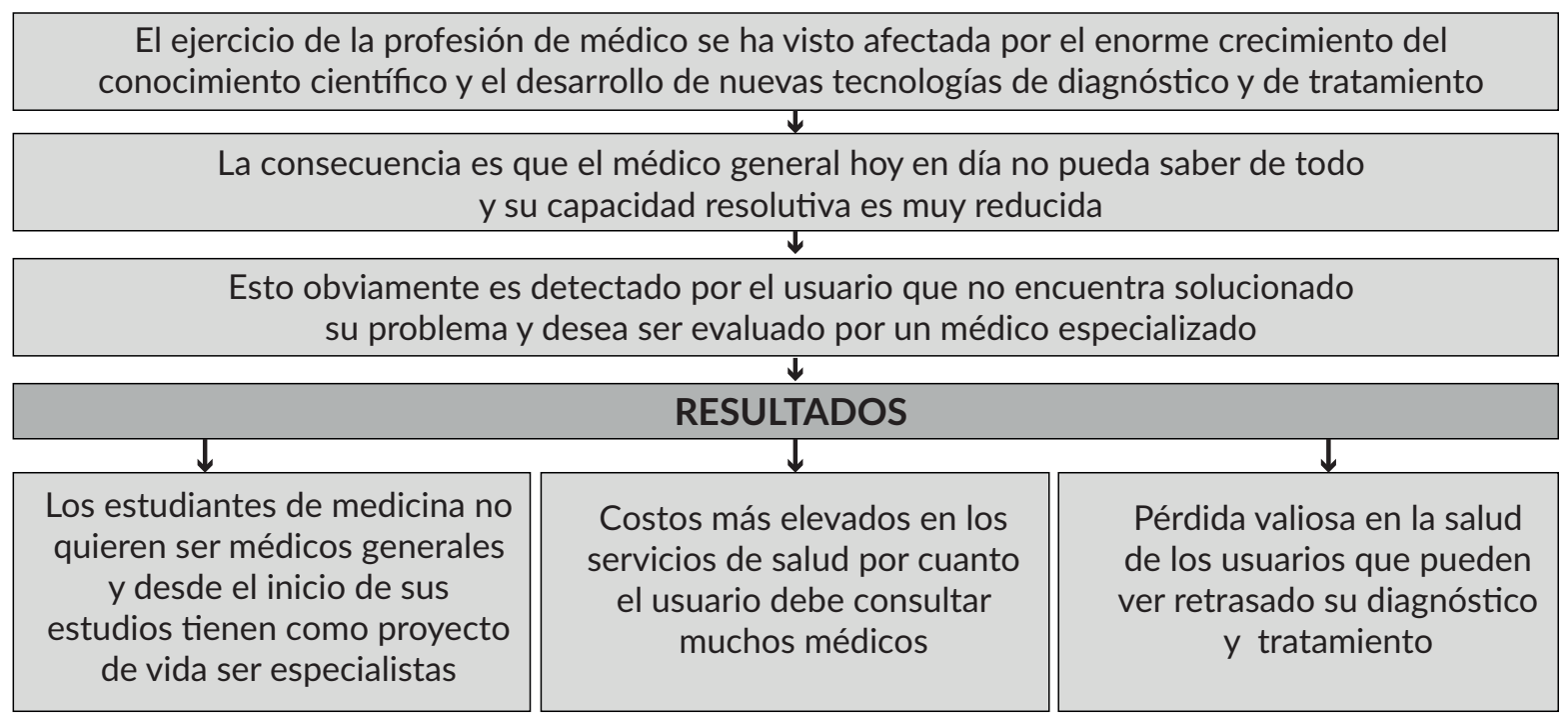

Fuente: elaboración propia basada en Ascofame (25), Bascuńan (52). 
El llamado "médico general" se ha convertido en Colombia sólo en un paso obligatorio para poder llegar a ser especialista, como lo demuestran múltiples investigaciones. En entrevistas con decanos de Facultades de Medicina, estudiantes, internos, médicos rurales y especialistas, se encuentra que el $95 \%$ de los estudiantes tienen ya seleccionada una determinada especialidad que quieren hacer y ninguno quiere ser médico general (53).

Al médico general que están graduando nuestras facultades se le está obligando a hacer cosas para las cuales no tiene el adiestramiento apropiado; por otra parte, no están recibiendo la formación requerida para el desempeńo de las funciones que deberían realizar (54).

La búsqueda de una especialización es tortuosa y puede durar muchos ańos, la mayoría de los médicos generales no logran especializarse, se calcula que uno de cada veinte médicos generales lo logra.

Los médicos generales que logran ingresar a un programa de especialización, tendrán que estudiar un promedio de 4 ańos más para obtener ese título.

Esto significa que, si un joven ingresa de 18 ańos a la universidad, será muy difícil que haya concluido la formación que desea (ser especialista) antes de los 30 ańos, esto con grandes consecuencias económicas para su familia y su entorno cercano.

La otra cara de la moneda la constituyen aquellos médicos generales que no logran ingresar a un programa académico de especialización, estos ejercerán una medicina que no desean y además en condiciones laborales, la mayoría de las veces, nada confortables. La satisfacción profesional de los médicos es piedra angular del buen funcionamiento de un sistema de salud y un médico que no puede cumplir sus ilusiones académicas, laborales y sociales no hará de manera adecuada su trabajo.

Pero, más grave aún, los pacientes cada día más, quieren que los atienda un especialista, en gran parte una posición también producto del entorno, pero especialmente de la globalización educativa, del internet y de la gran segmentación y diversificación de servicios que se ofrecen.

Es decir, los pacientes no quieren ser evaluados por un médico general, y los médicos no quieren ser médicos generales, ¿entonces qué sentido tiene seguir formando médicos generales bajo el modelo actual?

"Contrario al lejanísimo tiempo en que estudiar medicina casi garantizaba un futuro tranquilo, hoy los médicos generales tienen tantos dolores ypadecimientos que en todo el país ya se cuentan 17 sindicatos que los agremian"(55).

\section{PROPUESTAS DE CAMBIO}

Del pasado al presente se concluye que no se puede comparar el médico general del pasado con el médico general que forman las universidades hoy, por varias razones:

a. El estudiante que llega a las universidades hoy es muy diferente, desde el primer día de clase ya llega con la mente fijada en convertirse en un especialista. Es muy difícil pretender que alguien que llega con el sueńo de ser oftalmólogo aprenda a atender partos con calidad, pero peor aún, el ejercicio de este profesional, una vez graduado va a ser frustrante sino logra especializarse (45-50).

b. Los profesores de las universidades hoy en día son muy diferentes a los del pasado. Muy seguramente, hace 30 ańos, un profesor de oftalmología hacía parte de su ejercicio profesional como médico de familia y tenía un buen entrenamiento general. Hoy en día el porcentaje de profesores universitarios que son estrictamente médicos generales son mínimos, los cálculos más optimistas hablan del 7\%, los demás son profesores supra especializados en un área de la medicina.

c. No se puede comparar el ejercicio de la medicina dentro del sistema de salud existente en Colombia hoy, con el ejercicio que se daba en el pasado:

d. La ciencia ha aumentado exponencialmente, matemáticamente es imposible que hoy en día un médico sepa de todo, como se pretende que sea el médico general.

e. Las normas legales y éticas que existen hoy hacen que el profesional tenga que ser más cuidadoso con lo que hace. Muy seguramente un médico graduado hace 30 ańos realizaba cesáreas, eso es imposible hoy.

f. Los usuarios reclaman derechos y tienen más conocimiento o acceso a él. En épocas pasadas, ante el paciente el único que sabía era el médico, hoy en día, el médico receta un medicamento a un paciente, el paciente se va a consultar en internet y en media hora sabe tanto o más que el médico de ese medicamento.

g. Los usuarios hoy exigen especialistas, pero lo más interesante es que cada uno de nosotros como usuarios también. 
La causa de esta situación se atribuye a inoperancia de los sistemas de salud, pero es posible que sea, además, producto de la evolución natural de la sociedad $(56,57)$.

Durante el XXIII Congreso Colombiano de Medicina Interna en Cartagena, Colombia se plantearon las siguientes propuestas de orientación del médico general (58):

1. Dirigir la orientación al médico comunitario. Orientación frecuentemente rechazada por los estudiantes que sueńan con ser super especialistas (59).

2. Disminuir la intensidad de la formación en ciencias básicas. Las ciencias básicas cambian tan rápido que puede ser más productivo incrementar la docencia en áreas clínicas, involucrando el sustento básico en la clínica.

3. Concentrar la formación del médico general en entidades trazadoras. Si el médico general no puede aprender de todo, que aprenda bien lo que más requiere el sistema.

4. No formar más médicos generales. Se propone que las universidades desde que reciben un estudiante lo orienten a una especialidad (o un énfasis si se quiere). Ya son pocos los países desarrollados en el mundo donde todavía existen médicos generales, por ejemplo, en Estados Unidos y en Espańa se calcula que menos del 10\% de médicos ejercen como médicos generales $(60,62)$.

\section{PROPUESTA FINAL}

El mundo está preocupado por la formación de sus médicos y los efectos que esto implica sobre la salud de la comunidad y el buen desarrollo de los sistemas de salud estatales y privados (63).

La revisión del estado del arte permite concluir que existen bastantes elementos teóricos y sustentaciones investigativas que invitan a revisar la pertinencia del currículo actual en la satisfacción académica, laboral y social durante el ejercicio profesional de médicos generales en todo el mundo (64-68).

La evidencia parece hacer pensar que el médico general integral sabelotodo es cada día más utópico. Ya lo había dicho Rojas en 1991 (53), y es un hecho que muchas universidades han dado pasos en este sentido, algunas instituciones del país permiten hacer el internado como primer ańo de una especialización o permiten realizar la carrera con un énfasis. La Universidad de los Andes, por ejemplo, dice en la presentación de su programa: En los Andes no se gradúa un solo tipo de médico sino seis, dependiendo del área de escogencia del estudiante; de esta forma el egresado cuenta con diferentes posibilidades laborales (69). En la Universidad del Valle se permite realizar el último ańo de la carrera de medicina como primer ańo de la Especialización en Medicina Familiar.

La propuesta que invito a estudiar, retomando la de Rojas, es avanzar a la especialización de la medicina creando un programa de estudios que comprenda cuatro ańos de ciencias básicas y de salud pública; durante estos cuatro ańos se afianzarían conceptos de gran crecimiento en medicina como son la la anatomía, la fisiología, la biología molecular, la inmunología, la farmacología e igualmente se incrementaría la capacitación en programas de promoción de la salud y prevención de la enfermedad. En un quinto ańo el estudiante realizaría rotaciones cortas por todas las especialidades clínicas, en la mayoría de ellas como observador y en algunas quizá adquiriendo algunas competencias básicas que puedan ser de utilidad general (reanimación cardiopulmonar, manejo de líquidos y electrolitos) o ameritan un entrenamiento que puede lograrse en un tiempo breve (suturas, drenaje de abscesos, atención de un parto, etc.). A continuación, el estudiante realizaría cuatro ańos de profundización en un área o especialización en ciencias básicas, ciencias clínicas, ciencias quirúrgicas o de salud pública (Figuras 4 y 5).

De esta manera en un programa continuo, de alrededor de nueve o diez ańos, es posible formar especialistas jóvenes y de avanzar hacia doctorados o supra especializaciones (70).

Se invita a las directivas universitarias a analizar esta información y planear una oferta académica que contribuya a lograr las metas de oferta y demanda de talento humano en salud planteada por la política pública y cuyo debate académico es prioritario (71).

Es importante discutir y reflexionar sobre el tipo de médico que desean ser los estudiantes de medicinas y las necesidades sociales y del sistema de salud.

\section{CONFLICTOS DE INTERÉS Y FUENTES DE FINANCIACIÓN}

El autor declara que no existe conflicto de interés para la publicación del presente artículo. El artículo es producto del grupo de investigación Instituto de Investigaciones Biomédicas, Categoría A de Colciencias. No se requirió financiación específica para su desarrollo. 
Figura 4. Propuesta para un nuevo plan de estudios para la formación de médicos.

\begin{tabular}{|c|c|c|}
\hline Semestres 1 a 8 & Semestres 9 y 10 & Semestres 11 a 18 (20 en algunos) \\
\hline \multirow{2}{*}{$\begin{array}{l}\text { Ciencias básicas } \\
\text { médicas } \\
\text { (Biologia, Morfologia, } \\
\text { Bioquimica, Fisiología, } \\
\text { Genética, Farmacologia, } \\
\text { Inmunologia, etc) }\end{array}$} & \multirow{6}{*}{$\begin{array}{l}\text { Semestres de } 20 \\
\text { semanas para realizar } \\
\text { de manera rotatoria y } 12 \\
\text { semanas de vacaciones } \\
\text { al año: } \\
\text {-Historia clínica } \\
\text {-Examen físico general } \\
\text {-RCP } \\
\text {-Líquidos y electrolitos } \\
\text {-Pequeña cirugía } \\
\text {-Atención parto normal } \\
\text {-Atención niño sano } \\
\text {-Actividades de P\&P } \\
\text {-Introducción a la } \\
\text { psicoterapia } \\
\text {-Otros temas básicos }\end{array}$} & $\begin{array}{l}\text { Especialidades integradoras } \\
\text { (Especialista en atención primaria, en } \\
\text { cirugía básica, en medicina familiar, en } \\
\text { urgencias, en P\&P, etc.) }\end{array}$ \\
\hline & & $\begin{array}{l}\text { Especialidades clínicas } \\
\text { (Medicina interna, pediatria, psiquiatria, } \\
\text { físiatria, medicina deportiva, etc) }\end{array}$ \\
\hline \multirow{2}{*}{$\begin{array}{l}\text { Ciencias sociales y de } \\
\text { salud pública } \\
\text { (Antropología, Ecologia, } \\
\text { Sociología Médica, } \\
\text { Ética, Bioética, } \\
\text { Epidemiología, Salud } \\
\text { Pública, Promoción de } \\
\text { la salud, Prevención de } \\
\text { la enfermedad, etc) }\end{array}$} & & $\begin{array}{l}\text { Especialidades quirúrgicas } \\
\text { (Cirugia general, urologia, oftalmología, } \\
\text { ORL, plástica, ortopedia, neuro, etc) }\end{array}$ \\
\hline & & $\begin{array}{l}\text { Maestrías y doctorados en ciencias } \\
\text { básicas } \\
\text { (Biologia, Morfología, Bioquímica, } \\
\text { Genética, Fisiologia, Farmacologia, etc) }\end{array}$ \\
\hline \multirow{2}{*}{$\begin{array}{l}\text { Salud mental } \\
\text { (Psicobiología, } \\
\text { Psicopatología, } \\
\text { Comportamiento } \\
\text { humano, etc) }\end{array}$} & & $\begin{array}{l}\text { Maestrias y doctorados en salud pública } \\
\text { (epidemiología, administración, auditoria, } \\
\text { salud ocupacional, etc) }\end{array}$ \\
\hline & & $\begin{array}{l}\text { Otras } \\
\text { (Comunicación, informática médica, etc.) }\end{array}$ \\
\hline
\end{tabular}

Fuente: Rojas (53), Torres (61).

Figura 5. Propuesta de la nueva estructura curricular para la formación de un nuevo médico.

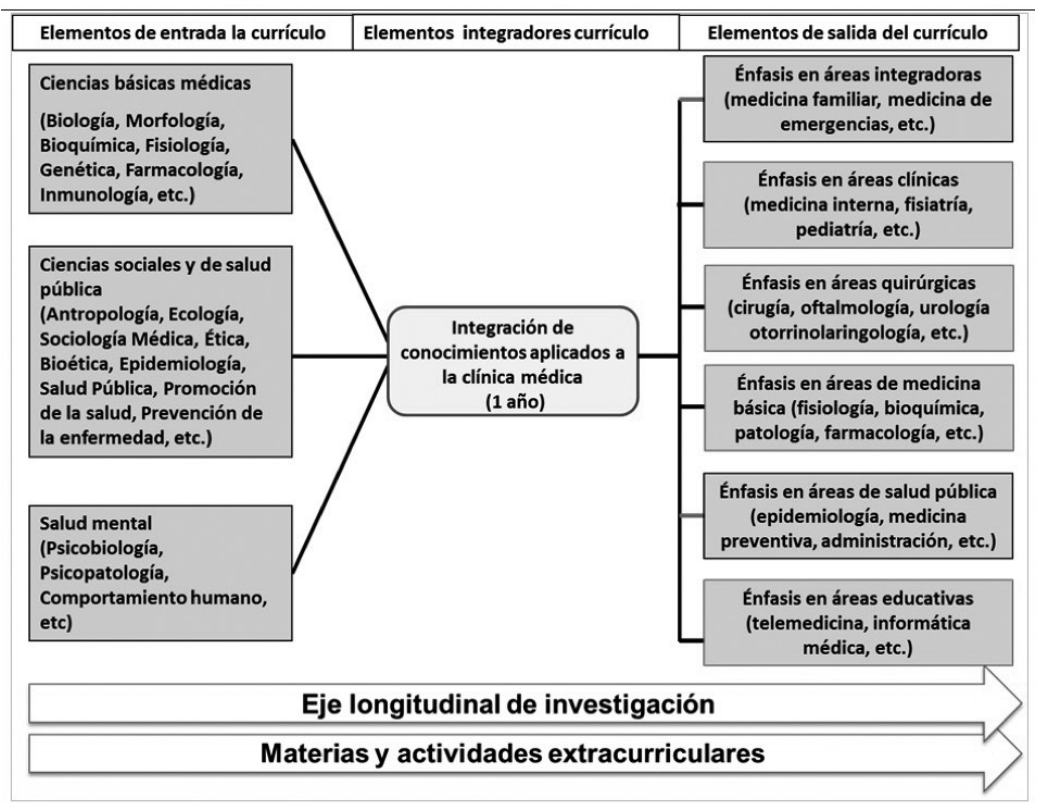

Fuente: Elaboración propia.

\section{REFERENCIAS}

1. Lain P. Antropología médica. 1 Ş ed. Barcelona: Salvat; 1984. p. 347-50.

2. Rojas W. Historia de la medicina. 1Ş ed. Medellín: CIB Fondo Editorial; 2012. p. 56-8.
5. Duffy T. The Flexner Report-100 Years Later. Yale J Biol Med. 2011; 84(3):269-76.

6. Byrn NR. Tendencias actuales de la educación médica y propuestas de orientación para la educación médica en América Latina. Educ Med Salud. 1993; 28:53-123.

7. Flexner A. An autobiography. 1 s ed. New York: Simon and Schuster, 1960. p. $72-8$

8. Flexner A. Medical Education in Europe: a report to the Carnegie Foundation for the advancement of teaching. 1Ş ed. Boston: D. B. Updike, The Meurymoukt Press.1912. p. 32-40.

9. Patińo J. Un currículo equilibrado. Trib Med.1993; 88:270-9.

10. Billroth T. The Medical Sciencies in the German Universities: with an introduction by William $\mathrm{H}$. Welch. New York: The Macmillan Company, 1924. p. 65-9

11. Universidad del Valle, Fondo Universitario Nacional. Memorias del Primer Seminario de Educación Médica en Colombia. 1Ş ed. Cali: Carvajal y Cía, 1957. p. 127-35

12. Departamento del Valle. Primer Congreso Departamental de Hospitales. 1Ş ed. Cali: Beneficencia del Valle, 1959. p. 113-43

13. Banta H. Flexner and medical education in Colombia. J Med Educ. 1972; 32: 879-85.

14. Flexner A. Medical Education in the United States and Canada: A Report to the Carnegie Foundation for the Advancement of Teaching. New York: Carnegie Bulletin, 1910. p. 364. Disponible en: http:// archive.carnegiefoundation.org/ pdfs/elibrary/Carn-egie_Flexner_ Report.pdf (consultado el 10 de enero de 2017)

15. González A. Plan para el desarrollo de las instituciones de salud. $1^{\mathrm{a}} \mathrm{ed}$. Cali: Carvajal y Cía, 1980. p. 225-37.

16. Cate 0. Formación médica y evaluación basadas en las competencias: ¿de qué se trata y 
cómo puede relacionarse con la práctica clínica? Rev Arg Cardiol. 2011; 79:405-9.

17. Noriega TO. La competencia clínica como eje integrador de los estudios de pre y posgrado en las ciencias de la salud. Rev Fac Med (Caracas). 2013; 26(1):17-21.

18. Reyes A. Enfoque curricular basado en competencias en la educación médica. Comunidad y Salud. 2010; 8(1): 58-64.

19. Pinilla A. Modelos pedagógicos y formación de profesionales en el área de la salud. Acta Med Colomb. 2011;36:204-18.

20. Organización Panamericana de la Salud. Gestión del desempeńo basado en competencias. Washington: OPS Serie Paltex para ejecutores de Programas de Salud No.42. 2000. p. 17-22

21. Gutiérrez M. El estado actual del currículo de medicina. Rev Med Herediana. 2008; 19(3): 85-6.

22. Martínez E. El core curriculum: un debate en la educación médica. Educación y educadores. 2006; 9(2):189-196.

23. Universidad del Valle. La crisis de la educación. Seminario Nacional sobre currículo. 1Ş ed. Cali: Editorial Univalle. 1993. p.47-52

24. Patińo J. Formación médico general integral: balance entre áreas básicas, clínicas y salud pública. Trib Med. 1991; 14:22-25.

25. Ascofame. Metodología para la docencia asistencia. 1Ş ed. Bogotá: Camargo Editores, 1987. p. 35-9

26. Patińo JC. Las Bases Moleculares de la Vida y la Enfermedad. 1Ş ed. Bogotá: Fundación OFA para el Avance de las Ciencias Biomédicas, 1980. p. 78-84

27. Petersdorf R. Medical Education: the process, students, teachers and patients. En Vevier Ch., 1Ş ed. Flexner: 75 Years Later : a Current Commentary on Medical Education. Maryland: University Press of America, 1987; p. 17-22

28. Venturelli J. Educación médica: nuevos enfoques, metas y métodos. Washington: OPS/OMS Serie Paltex. 1987. p. 23-7

29. Venturelli J. Modernización de la educación médica: ¿Illusiones inútiles o necesidad imperiosa? Washington: OPS/OMS Serie Paltex. 2003. p. 76-8

30. Patińo J. La educación médica para el ańo 2000. Memorias de la Conferencia Panamericana de Enseńanza de la Demografía en las Facultades de Medicina. Bogotá: Ascofame. 1970.

31. Patińo J. Educación médica en el ańo 2000. Conferencia Andina de Educación Médica. Cartagena: Ascofame, 1993. p. 34

32. Patińo J. Educación Médica en el ańo 2000. Trib Med. $1993 ; 88: 234-48$

33. Kane M. The assessment of professional competence. Education and the Health Professions. 1992; 15:163-182.

34. Castańeda 0. El papel del liderazgo en la gestión médica. Aten Fam. 2015;22(1):1.
35. Patińo J. La desprofesionalización de la medicina en Colombia. Acta Med Colomb. 2001; 26:43-4.

36. Narváez AI. Sarmiento GJ. Habilidades clínicas en la formación del médico general. Rev Fac Cienc Salud Univ Cauca. 2005; 7:8-18

37. Frank JD. The CanMEDS iniciative: implementing an outcomes-based framework of phisycian competences. Medical Teachers. 2007; 29: 642-647.

38. Frank J. CanMEDs 2015 Physician Competency Framework. Ottawa: Royal College of Physicians and Surgeon of Canada. 2015. p.102-10

39. Proyecto CANMEDS 2000. Habilidades necesarias en el nuevo milenio: Informe del grupo de trabajo sobre necesidades sociales. Real Colegio de Médicos y Cirujanos de Canadá. Canadá: Royal College of Physicians and Surgeons of Canada, 1996.

40. Bligh C. PRISMS. South East Asian J Med Educ. 2010; 4:1.

41. Burrow G. La medicina y la educación médica en el siglo XXI. Trib Med. 1993; 88: 217-21.

42. Jiménez A. Identificación de los diez principales problemas de la educación médica en Colombia. Memorias del Encuentro sobre Educación Médica, Asociación Colombiana de Facultades de Medicina, Bogotá, 1987. p. 34.

43. Maldonado J. ¿Necesita Colombia más médicos o mejor preparados? Iladiba, 2011; 360:2-3.

44. Torres C. Médicos generales: ¿Una respuesta al pasado? Rev Col Salud Libre. 2008; 3:157-63.

45. Torres C. Especialidades preferidas por estudiantes de dos Escuelas de Medicina de Cali Colombia. Rev Col Salud Libre. 2009; 4:45-54.

46. Torres, C. Ramírez, M.P. Ospina, J. Díaz, V. Expectativas para el ejercicio profesional de la medicina en estudiantes que inician su carrera universitaria. Inv Ed Med. 2017;6(23):190-97.

47. Toso A. Intereses y perspectivas sobre la carrera de medicina: un contraste entre estudiantes de medicinan de primero y séptimo ańos. Rev Méd Chile. 2012; 140:609-15.

48. Bassan N. Médico general o especialista. Tendencia en alumnos de $1^{\circ}$ y $5^{\circ}$ ańos de la Escuela de Medicina de Rosario de la Universidad Abierta Interamericana (UAI). Rev Educ Cienc Salud 2008; 5(2):108-12.

49. Breinbauer HGF. Tendencia en el estudiante medicina a ejercer como médico general o especialista. Rev Med Chile, 2009; 137:865-72.

50. Marín E. El médico general, una respuesta al futuro. En Ascofame, Memorias seminario El médico general, una respuesta al futuro. Bogotá. 1978. p. 123-6

51. Echeverri DY. ¿dónde está el paciente? Rev Col Cardiol $2016 ; 23: 253-4$ 
52. Bascuńán L. Cambios en la relación médico-paciente y nivel de satisfacción de los médicos. Rev Med Chile, 2005; 133: 11-21.

53. Rojas W. Reflexiones sobre la educación médica en Colombia. Rev Col Cirugía, 1991; 6:125-9.

54. Rojas W. Educación y atención médica. Acta Med Colomb 2009; 34:16-20.

55. Rojas J. Testimonios dolorosos: ser médico medio de la crisis de la salud. Cali. Diario El País, 2017. Disponible en: http://www.elpais.com.co/elpais/cali/noticias/testimoniosdoloroso (Consultado en noviembre 20 de 2017)

56. Gómez VM. Hacia la diferenciación y la especialización en la educación superior. Propuesta para el caso de Colombia. Educ Superior Sociedad. 2015; 2:112-27

57. Gawande, A. The checklist manifiesto. 1S ed. New York: Picador Ed. 2011.

58. Simposio del XXIII Congreso Colombiano de Medicina Interna en Cartagena, Colombia. 2014.

59. García-Huidibro D. Expectativas de estudiantes de medicina de pregrado en relación con el perfil del médico esperado. Rev Med Chile. 2006; 134: 947-54.

60. Centro de Proyección para el desarrollo. Estudio de disponibilidad y distribución de la oferta de médicos especialistas, en servicios de alta y mediana complejidad en Colombia. Informe final. Bogotá: Universidad Javeriana. 2013.

61. Torres C. Propuesta para la especialización del ejercicio de la medicina en Colombia. Salud Cultura. 2014; 5:21-26.

62. Roselli DA. La medicina especializada en Colombia. Una aproximación diagnóstica. Bogotá: Centro Editorial Javeriano. 2000.

63. Scheurer DM. U.S. Physician Satisfaction: A Systematic Review. J Hosp Med. 2009; 4(9): 560-70.

64. Pinilla A, Parra G, Hernández H. Propuesta curricular para la formación de médicos internistas en Colombia. Acta Med Colomb 2016; 41:248-58.

65. Sendoya SY. Nivel de satisfacción de la médica egresada de la Universidad del Valle, Cali. Colomb Med 2000; 31:118-121.

66. Toro-Huamanchumo CJ. Satisfacción laboral del médico egresado de una universidad peruana: estudio preliminar. Inv Ed Med. 2017; disponible en http:// dx.doi.org/10.1016/j.riem.2017.01.151

67. Huamán M, Kamimura K, Medina J, Bustíos C, Miní E, Benito M, et al. Características laborales del médico joven egresado de la Facultad de Medicina San Fernando. An Fac Med. 2007; 68:19-28.

68. De Oliveira P, de Souza MR, Elias PEM, D'Ávila AL. Physicians' job satisfaction and motivation in a public academic hospital. Hum Resour Health. 2016; 14:75-8.

69. Medicina.uniandes.edu.co/programas/pregrado [Internet]. Bogotá: Universidad de los Andes, [citado 15 mayo 2018].
Universidad de los Andes, Facultad de Medicina, pregrado; [1 p.] Disponible en https://medicina.uniandes.edu.co/ index.php/es/programas/pregrado

70. Mayol J. Debe plantearse un grado de dos ańos. Rev Médica. 2015; 43:30-32.

71. Consejo de Facultad Ciencias de la Salud Universidad del Cauca. Recomendaciones para la transformación de la educación médica en Colombia. Rev Fac Cienc Salud Univ Cauca. 2017; 19(1):12-15. 